\title{
Detached binaries in the Large Magellanic Cloud
}

\section{A selection of binaries suitable for distance determination ${ }^{\star}$}

\author{
G. Michalska and A. Pigulski
}

\author{
Instytut Astronomiczny Uniwersytetu Wrocławskiego, Kopernika 11, 51-622 Wrocław, Poland \\ e-mail: [michalska;pigulski]@astro.uni.wroc.pl
}

Received 9 November 2004 / Accepted 3 December 2004

\begin{abstract}
As a result of a careful selection of eclipsing binaries in the Large Magellanic Cloud using the OGLE-II photometric database, we present a list of 98 systems that are suitable targets for spectroscopic observations that would lead to the accurate determination of the distance to the LMC. For these systems we derive preliminary parameters combining the OGLE-II data with the photometry of MACHO and EROS surveys. In the selected sample, 58 stars have eccentric orbits. Among these stars we found fourteen systems showing apsidal motion. The data do not cover the whole apsidal motion cycle, but follow-up observations will allow detailed studies of these interesting objects.
\end{abstract}

Key words. stars: binaries: eclipsing - Magellanic Clouds - stars: fundamental parameters

\section{Introduction}

Diverse methods have been employed in the last decade to derive distances to the Magellanic Clouds (see, e.g., Cole 1998; Harries et al. 2003; Alves 2004). The knowledge of these distances is important for at least two reasons. First, Magellanic Clouds play a key role in the calibration of the Cepheid-based distance scale. Second, due to the low metallicity of the Clouds, it can be checked how different methods used for distance determination depend on metallicity. Unfortunately, as far as the distance moduli of the Magellanic Clouds are concerned, different methods gave results that disagree at a level that leaves in doubt their applicability for more distant objects.

However, the idea of measuring distances by means of eclipsing binaries seems very promising and supposedly will soon succeed in finding the correct answer. The method has been known for many years and first applied to a Magellanic Cloud star by Bell et al. (1991). Its potential usefulness has been recalled a few years ago by Paczyński (1997), while historical outlook was presented by Kruszewski \& Semeniuk (1999). The method exploits the advantages of a combination of the light curve of an eclipsing binary with its double-lined spectroscopic orbit. This combination provides radii, masses and ratio of surface brightnesses of the components. To get the distance, it is enough to calibrate surface brightness, $F_{\mathrm{V}}$, in terms of an easily-observed colour index and account for interstellar extinction. The calibration of $F_{\mathrm{V}}$ is usually made

^ Table 3 is only available in electronic form at the CDS via anonymous ftp to cdsarc.u-strasbg.fr (130.79.128.5) or via http://cdsweb.u-strasbg.fr/cgi-bin/qcat?]/A+A/434/89 using nearby (that is, having accurate parallaxes) eclipsing binaries and/or interferometric and occultation radii of stars (Barnes et al. 1978; di Benedetto 1998; van Belle 1999).

In order to avoid possible systematic effects on the calibration of $F_{\mathrm{V}}$ for close binaries, it was pointed out at the beginning that detached systems (hereafter DEB) are the best for the purpose of distance determination (e.g. Paczyński 1997). However, since alternatively to the application of the calibration of $F_{\mathrm{V}}$, the fit of the modern model atmospheres to the UV/optical spectrum can be utilized, the advantage of using only the DEBs is not so straightforward. As was argued by Wyithe \& Wilson (2002) and Wilson (2004), semi-detached binaries can be used for the purpose of distance determination as well. Unlike in the DEBs, the photometric mass ratio can be derived, so that a single-lined spectroscopic orbit is sufficient. In addition, current modeling programs describe the proximity effects very well. Moreover, the orbits of semi-detached systems are well circularized, which is not always the case for the DEBs. In fact, out of ten eclipsing systems used by Harries et al. (2003) to get the distance to the Small Magellanic Cloud (SMC), only three are the DEBs, while six have a semi-detached geometry. Since each binary provides the distance, one can get the average distance with an unprecedented accuracy using as many systems as possible. The ten systems analyzed by Harries et al. (2003) led to the - so far - best estimation of the SMC distance modulus: $18.89 \pm 0.04 \mathrm{mag}$ with a 0.10 -mag uncertainty due to systematic effects. The authors, however, intend to derive the distance of the SMC using over 100 eclipsing binaries.

A similar work is underway for the Large Magellanic Cloud (hereafter LMC). So far, seven systems were used to get its 
distance. Out of them, only three, HV 2274 (Guinan et al. 1998; Udalski et al. 1998a; Ribas et al. 2000; Nelson et al. 2000; Groenewegen \& Salaris 2001; Fitzpatrick et al. 2002), EROS 1044 (Ribas et al. 2002) and HV 982 (Fitzpatrick et al. 2002; Clausen et al. 2003), are DEBs. Out of the remaining four, three, i.e. HV 5936 (Bell et al. 1993; Fitzpatrick et al. 2003), HV 2241 (Pritchard et al. 1998; Ostrov et al. 2001) and HV 2543 (Ostrov et al. 2000) are semi-detached systems, whereas Sk $-67^{\circ} 105$ (Ostrov \& Lapasset 2003) is even a contact binary. As indicated above, in order to derive the distance accurately, we need to analyze many more systems.

Fortunately, microlensing surveys delivered photometric measurements for millions of stars in the Clouds and catalogs including thousands of eclipsing binaries were published. First, Grison et al. (1995) published double-band photometry for 79 eclipsing systems in the LMC bar discovered within the EROS survey. Next, Alcock et al. (1997) classified 611 eclipsing binary stars in the LMC from the MACHO survey. Finally, the catalogs of eclipsing binaries from the OGLE-II survey were published: for the SMC (Udalski et al. 1998b; Wyrzykowski et al. 2004), and the LMC (Wyrzykowski et al. 2003, hereafter W03). In total, about 4500 eclipsing binaries (1914 in the SMC and 2580 in the LMC) were found in the OGLE-II data in both Clouds. Therefore, the first step towards the accurate distance determination to the LMC is good selection of binaries that are suitable for this purpose.

This is the main goal of this paper. Using the photometry obtained during the second phase of the OGLE survey, OGLE-II, we have selected 98 DEBs brighter than 17.5 mag in $V$. We have also analyzed their light curves by means of the Wilson-Devinney (Wilson \& Devinney 1971; Wilson 1979, 1990) program, combining the photometry from the OGLE-II, MACHO and EROS surveys. The preliminary results of this paper have been presented by Michalska \& Pigulski (2004). A list of 36 DEBs in the LMC good for distance determination was presented by W03. A similar selection has been done for the SMC eclipsing binaries by Udalski et al. (1998b), Wyithe \& Wilson (2001), and Graczyk (2003).

The data used in this study are described in Sect. 2. Section 3 presents our selection criteria followed by description of the new transformation of the OGLE-II differential fluxes to magnitudes (Sect. 4). The analysis of the light curves and discussion of the parameters is described in Sect. 5. Finally, we discuss systems with apsidal motion (Sect. 6) and provide our conclusions (Sect. 7).

\section{The photometry}

\subsection{OGLE data}

The OGLE observations we used were carried out during the second phase of this microlensing survey (Udalski et al. 1997) with the 1.3-m Warsaw telescope at the Las Campanas Observatory, Chile. They cover about 4.5 square degrees in the LMC bar (twenty one $14^{\prime} \times 57^{\prime}$ fields). The data span almost four years (1997-2000). This was the primary source of our photometric data used to select the DEBs (Sect. 3). The other databases (MACHO and EROS) were used as supplementary ones.

The OGLE-II photometry for the LMC fields is currently available in three different forms:

- Mean BVI magnitudes for $\sim 7 \times 10^{6}$ stars (Udalski et al. 2000) derived by means of the profile-fitting package DoPHот (Schechter et al. 1993).

- Time-series $I$-filter photometry of about 53400 variable candidates published by Żebrun et al. (2001b). The photometry was obtained by means of the Difference Image Analysis (DIA) package developed by Woźniak (2000) which is an implementation of the image subtraction method of Alard \& Lupton (1998). The photometry is available from the OGLE web page ${ }^{1}$ in two forms: as differential fluxes and the magnitudes transformed from these fluxes. The transformation was explained in detail by Żebrun et al. (2001b).

- BVI time-series photometry for the same variable candidates obtained with DoPHOT and available from the same web page. Typically, about 30, 40 and 400 datapoints are available for each star in $B, V$ and $I$ bands, respectively.

Since the OGLE data were made in the drift-scan mode (Udalski et al. 1997), for a given frame the average epoch of observation is not the same for all stars. Following the prescription given by Żebruń et al. (2001b), we applied appropriate corrections. Moreover, the data were phased with the derived orbital period and the outliers were rejected.

\subsection{MACHO data}

The MACHO observations were obtained in blue (440-590 nm) and red (590-780 nm) bands, rougly coincident with Johnson $V$ and Cousins $R$, respectively. The telescope used was the 1.27-m Great Melbourne Telescope situated at Mount Stromlo, Australia. The MACHO data span almost eight years between 1992 and 2000. The photometry is available through the MACHO web page (Allsman \& Axelrod 2001).

The MACHO data are affected by the presence of a large number of outliers. We therefore rejected them in an iterative process fitting accurately the shape of the light curve in the phase diagram. In addition, a spurious variation of instrumental origin with a 1-yr period was removed prior to the analysis. Then, the heliocentric corrections were applied to the published epochs. Since the latter corresponded to the beginning of exposures, half the exposure time (150 s) was added to the epochs as well.

\subsection{EROS data}

The EROS observations were made in 1991-1992 with the 0.4-m telescope at La Silla, Chile, in two bands, $B_{\mathrm{E}}$ and $R_{\mathrm{E}}$, having central wavelengths of 490 and $670 \mathrm{~nm}$, respectively (Grison et al. 1995). Since the latter corresponds roughly to the MACHO red band, we used in our study the photometry made

${ }^{1}$ http://www.sirius.astrouw.edu.pl/ ogle/ogle2/dia/ 
in the $B_{\mathrm{E}}$ band only. The epochs of EROS data were published in local time (see Ribas et al. 2002), so that a three-hour correction was added. As for the OGLE and MACHO data, some outliers were removed prior to analysis.

\section{Selection of objects}

As we pointed out in previous sections, the OGLE-II database of candidate variables (Żebrun et al. 2001b) was the primary source of the analyzed data and the subject of the main selection process. From this catalog, we first extracted the $I$-filter photometry of those stars that were brighter than 17.5 mag in $V$ and had $V-I<0.5 \mathrm{mag}$ (Fig. 1). For all these stars, the analysis of variance (AoV) periodograms of Schwarzenberg-Czerny (1996) were calculated. Then, the data were phased with the period corresponding to the frequency of the maximum peak in the periodogram and examined visually. After this check, the star was selected for further analysis if: (i) the light curve indicated it was an EA-type eclipsing binary, presumably a DEB; (ii) the proximity effects were small; (iii) the scatter in the light curve was relatively low.

In total, we found 98 stars that meet our three criteria. For all but five the MACHO photometry is available, eleven are in the list of eclipsing binaries found within the EROS survey. The stars are listed and cross-identified in Table 1.

Our selection was made independently of the work of W03, who - using the same data - discovered 2580 eclipsing binaries, including 1817 of the EA type. It was, however, not our aim to make a complete catalog of eclipsing binaries in the LMC, but to select the brightest DEBs with the best photometry. There are 403 EA-type eclipsing binaries in the catalogue of W03 that fall into the selection box in Fig. 1. Thus, the stars we selected constitute about $25 \%$ of this sample. However, three objects from our list, \#24, \#87, and \#912, were not included in the W03 catalogue.

\section{New transformation to magnitudes}

As mentioned in Sect. 2.1, two sources of time-series data in the $I$ filter expressed in magnitudes are available from the OGLE-II survey. At the beginning, we had to decide which of them use in the subsequent analysis. Due to the crowded nature of the LMC fields, the DIA photometry has considerably smaller scatter than that obtained by means of profile fitting and hence it seems to be preferable. However, this photometry suffers from the unavoidable bias occuring during the transformation from differential fluxes to magnitudes (see, e.g., Woźniak 2000; or Żebruń et al. 2001a). The bias in the transformed magnitude, $m_{\text {DIA }}$, comes mainly from the uncertainty of the reference flux, $f_{\text {ref }}$, in the transformation equation:

$m_{\text {DIA }}=-2.5 \log \left(f_{\text {ref }}+\Delta f\right)+C$.

In the above equation, $\Delta f$ denotes differential flux, and $C$ is the zero point of the magnitude scale. While the uncertainty in $C$ does not affect the shape and the magnitude range of the

\footnotetext{
2 The numbers preceded by "\#” refer to the first column of Table 3, available only in electronic form from the CDS.
}

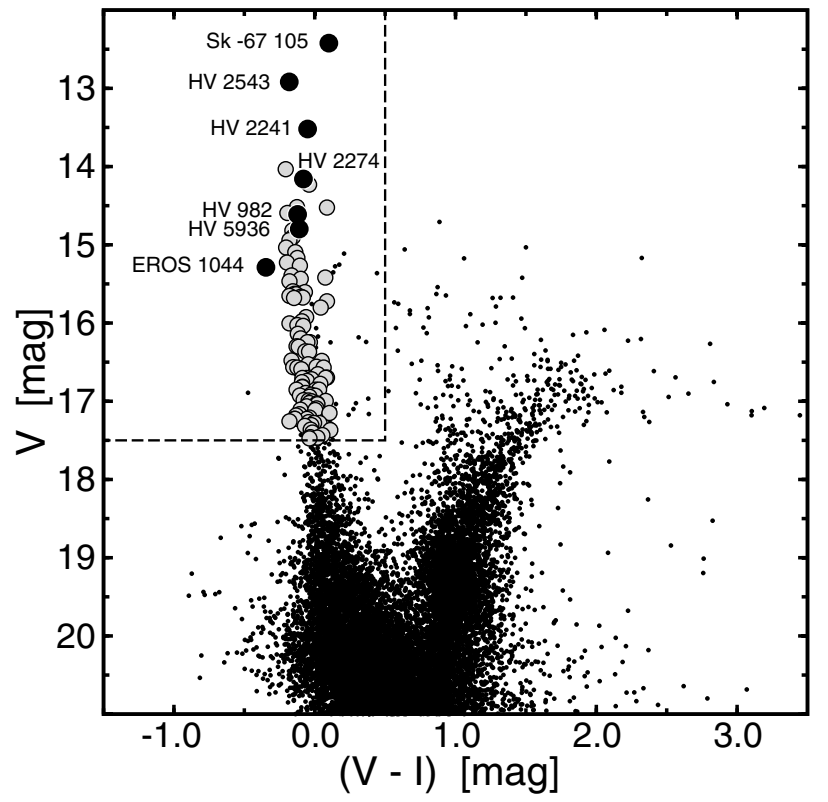

Fig. 1. The colour-magnitude diagram for $\sim 16000$ stars from the OGLE-II SC_1 field (small dots). The 98 DEBs we selected as good targets for distance determination are plotted as gray filled circles. For comparison, seven eclipsing binaries already used for the determination of the distance to the LMC are plotted as black filled circles and labeled. The dashed line delimits our selection box.

transformed light curve, that of $f_{\text {ref }}$ does. In consequence, the parameters derived from the fit of the light curve would be affected as well.

The problem is illustrated in Fig. 2 for a star that was observed within OGLE-II in two overlapping fields. The top panel shows the light curve derived by means of DoPнот. The light curves from the two fields agree quite well, there is only a small 0.003-mag shift in the mean brightness between them. On the other hand, the DIA light curves (middle panel) are less scattered, but one is shifted by $\sim 0.1 \mathrm{mag}$ with respect to the other and the eclipses have slightly different depths. This is because $f_{\text {ref }}$ was derived merely from the profile-fitting in the reference images. The reference images, although obtained by co-adding 20 best frames in each field (Żebruń et al. 2001a), were still quite crowded. Therefore, we calculated new $f_{\text {ref }}$ and $C$ values in Eq. (1), fitting the DIA differential fluxes to the DoPHOT magnitudes by means of least-squares fits. The result is shown in Fig. 2c. This approach combines the advantages of both photometries: small scatter of the DIA photometry and the correct range in brightness of the DoPHOT one. A new transformation was derived for all eclipsing binaries selected for further analysis. Although the example shown in Fig. 2 is an extreme rather than a typical case, we encourage all users of the DIA photometry to do a new transformation in all applications where the correct magnitude scale is required.

\section{Analysis of light curves}

The light curves in three (or four, if the EROS $B_{\mathrm{E}}$ photometry was available) bands, MACHO blue and red, (designated thereafter $V_{\mathrm{M}}$ and $R_{\mathrm{M}}$, respectively) and OGLE $I$, were 
Table 1. List of 98 selected DEBs in the Large Magellanic Cloud. $P_{\text {orb }}$ stands for the orbital period. The VI photometry was derived from the OGLE-II DoPHOT data and is given for the out-of-eclipse phase at maximum light. In the last column "E" was given for system with non-zero eccentricity and "AM" for systems with detectable apsidal motion. For systems that were indicated as good targets for distance determination by W03, the number is followed by an asterisk. Stars are arranged according to the decreasing $V$ magnitude.

\begin{tabular}{|c|c|c|c|c|c|c|c|c|}
\hline \# & $\begin{array}{l}\text { OGLE } \\
\text { field(s) }\end{array}$ & $\begin{array}{c}\text { OGLE } \\
\text { name }\end{array}$ & $\begin{array}{c}\text { MACHO } \\
\text { name }\end{array}$ & $\begin{array}{l}\text { EROS } \\
\text { name }\end{array}$ & $\begin{array}{c}P_{\text {orb }} \\
{[\mathrm{d}]}\end{array}$ & $\begin{array}{c}V \\
{[\mathrm{mag}]}\end{array}$ & $\begin{array}{l}V-I \\
{[\mathrm{mag}]}\end{array}$ & Remarks \\
\hline 1 & LMC_SC13 & $05065201-6825466$ & 19.4302 .319 & & 6.33000 & 14.036 & -0.207 & $\mathrm{E}, \mathrm{AM}$ \\
\hline $2 *$ & LMC_SC2 & $05303928-7014097$ & 7.8147 .14 & & 24.673107 & 14.231 & -0.041 & $\mathrm{E}$ \\
\hline $3 *$ & LMC_SC11 & $05082813-6848251$ & 1.4539 .37 & & 2.995464 & 14.520 & -0.125 & $\mathrm{E}, \mathrm{AM}$ \\
\hline $4^{*}$ & LMC_SC17 & $05381862-7041084$ & 11.9351 .15 & & 2.191363 & 14.523 & 0.087 & \\
\hline $5^{*}$ & LMC_SC7 & $05180481-6948189$ & 78.6097 .13 & & 3.107029 & 14.591 & -0.193 & $\mathrm{E}$ \\
\hline $6^{*}$ & LMC_SC11 & 05092929-6855028 & 79.4779 .34 & & 2.678832 & 14.819 & -0.159 & HV 5622, E, AM \\
\hline $7 *$ & LMC_SC16 & $05351775-6943187$ & 81.8881 .47 & & 3.881843 & 14.902 & -0.156 & $\mathrm{E}, \mathrm{AM}$ \\
\hline $8^{*}$ & LMC_SC1/16 & $05350218-6944178$ & 81.8881 .44 & & 2.989472 & 14.941 & -0.178 & $\mathrm{E}$ \\
\hline $9 *$ & LMC_SC13 & $05063443-6825442$ & 19.4302 .345 & & 2.154488 & 15.036 & -0.202 & \\
\hline $10^{*}$ & LMC_SC7 & $05182818-6937453$ & 78.6220 .60 & 1017 & 1.403789 & 15.094 & -0.139 & \\
\hline $11 *$ & LMC_SC2 & 05322529-6925374 & - & & 3.370160 & 15.167 & -0.122 & $\mathrm{E}$ \\
\hline $12 *$ & LMC_SC6 & $05210081-6929449$ & 78.6585 .50 & & 1.300791 & 15.227 & -0.196 & \\
\hline $13^{*}$ & LMC_SC15 & $05014027-6851060$ & 1.3449 .27 & & 4.034834 & 15.267 & -0.105 & $\mathrm{E}, \mathrm{AM}$ \\
\hline $14^{*}$ & LMC_SC5 & $05224434-6931435$ & 78.6827 .52 & & 2.150539 & 15.391 & -0.166 & \\
\hline 15 & LMC_SC18 & $05404706-7036564$ & 11.9836 .21 & & 7.087663 & 15.420 & 0.075 & \\
\hline $16^{*}$ & LMC_SC9 & $05134140-6932455$ & - & & 5.457320 & 15.437 & -0.099 & $\mathrm{E}$ \\
\hline $17 *$ & LMC_SC5/6 & $05223546-6931434$ & 78.6827 .66 & 1036 & 2.183363 & 15.469 & -0.178 & \\
\hline $18^{*}$ & LMC_SC7 & $05185897-6935495$ & 78.6221 .90 & 1074 & 9.144018 & 15.596 & -0.155 & $\mathrm{E}$ \\
\hline $19 *$ & LMC_SC16 & $05371417-7020015$ & 11.9235 .33 & & 3.256696 & 15.606 & -0.069 & HV 5963, E \\
\hline $20^{*}$ & LMC_SC8 & $05164453-6932333$ & 78.5859 .100 & 1066 & 5.603540 & 15.624 & -0.143 & $\mathrm{E}$ \\
\hline $21^{*}$ & LMC_SC10 & $05102875-6920480$ & 5.4894 .3904 & & 3.773443 & 15.632 & -0.133 & $\mathrm{E}, \mathrm{AM}$ \\
\hline 22 & LMC_SC8 & $05170507-6945234$ & 78.5977 .2715 & & 2.864425 & 15.653 & -0.180 & $\mathrm{E}$ \\
\hline $23^{*}$ & LMC_SC11 & $05093433-6854259$ & 79.4779 .81 & & 1.462906 & 15.663 & -0.100 & \\
\hline 24 & LMC_SC13 & $05064333-6836115$ & 19.4300 .349 & & 4.018698 & 15.672 & -0.084 & $\mathrm{E}, \mathrm{AM}$ \\
\hline 25 & LMC_SC5 & $05225771-6935094$ & 78.6947 .2732 & & 10.187024 & 15.680 & -0.146 & \\
\hline $26^{*}$ & LMC_SC18 & 05410194-7005047 & 76.9844 .26 & & 2.385189 & 15.722 & 0.086 & \\
\hline $27 *$ & LMC_SC9 & $05132398-6922492$ & 5.5377 .4567 & & 2.636570 & 15.804 & 0.043 & $\mathrm{E}$ \\
\hline 28 & LMC_SC10 & $05110289-6913098$ & 79.5017 .83 & & 2.152919 & 15.923 & -0.061 & \\
\hline 29 & LMC_SC6/7 & $05195816-6928239$ & 78.6465 .173 & 1012 & 1.338312 & 15.979 & -0.092 & \\
\hline 30 & LMC_SC4 & $05251673-6929039$ & 77.7312 .90 & & 3.288241 & 16.005 & -0.178 & \\
\hline 31 & LMC_SC1 & $05345582-6943100$ & 81.8881 .97 & & 1.601593 & 16.028 & -0.122 & \\
\hline 32 & LMC_SC11 & $05081572-6929044$ & 5.4529 .1362 & & 2.899285 & 16.039 & -0.081 & HV 5619 \\
\hline 33 & LMC_SC9 & $05133633-6922416$ & 5.5377 .4656 & & 2.108729 & 16.140 & -0.119 & $\mathrm{E}, \mathrm{AM}$ \\
\hline 34 & LMC_SC5 & $05233948-6943346$ & 77.7066 .333 & & 2.145747 & 16.194 & -0.100 & \\
\hline 35 & LMC_SC15 & $05000218-6931561$ & 17.3197 .781 & & 3.317560 & 16.245 & -0.034 & $\mathrm{E}$ \\
\hline 36 & LMC_SC11 & $05095412-6853046$ & 79.4780 .100 & & 16.930492 & 16.247 & -0.051 & $\mathrm{E}$ \\
\hline 37 & LMC_SC14 & $05050424-6857588$ & 1.4052 .2379 & & 9.844484 & 16.299 & -0.130 & $\mathrm{E}$ \\
\hline 38 & LMC_SC8 & $05152057-6852502$ & 79.5627 .88 & & 1.538050 & 16.301 & -0.113 & \\
\hline 39 & LMC_SC4/5 & $05250946-7004226$ & 77.7303 .152 & & 3.625522 & 16.364 & -0.066 & $\mathrm{E}$ \\
\hline 40 & LMC_SC18 & $05404159-6959014$ & 76.9845 .63 & & 2.009981 & 16.367 & -0.040 & $\mathrm{E}$ \\
\hline 41 & LMC_SC6 & $05221179-6928551$ & 78.6828 .213 & & 7.544703 & 16.479 & -0.166 & $\mathrm{E}$ \\
\hline 42 & LMC_SC5 & $05250140-6955086$ & 77.7184 .162 & & 8.013626 & 16.482 & 0.050 & $\mathrm{E}$ \\
\hline 43 & LMC_SC15 & $05010991-6904496$ & 18.3325 .230 & & 9.771474 & 16.525 & -0.045 & $\mathrm{E}$ \\
\hline 44 & LMC_SC19 & $05425713-7009580$ & 76.10205 .471 & & 7.229139 & 16.557 & 0.014 & $\mathrm{E}$ \\
\hline 45 & LMC_SC6 & $05213496-6925346$ & 78.6708 .180 & & 2.598897 & 16.565 & -0.152 & $\mathrm{E}, \mathrm{AM}$ \\
\hline 46 & LMC_SC2 & $05315853-6955320$ & - & & 2.918065 & 16.567 & 0.063 & $\mathrm{E}$ \\
\hline 47 & LMC_SC13 & $05053815-6820531$ & 19.4062 .914 & & 5.621099 & 16.569 & -0.122 & $\mathrm{E}$ \\
\hline 48 & LMC_SC13 & $05072467-6829325$ & 19.4423 .464 & & 1.672507 & 16.589 & -0.095 & \\
\hline 49 & LMC_SC9 & $05130842-6908018$ & 79.5260 .94 & & 1.240540 & 16.655 & 0.022 & \\
\hline 50 & LMC_SC10 & 05121954-6914547 & 79.5137.189 & & 8.523814 & 16.697 & 0.086 & $\mathrm{E}$ \\
\hline 51 & LMC_SC4 & $05265371-6959493$ & 77.7546 .303 & & 2.431105 & 16.700 & 0.077 & \\
\hline 52 & LMC_SC4 & $05263667-6951253$ & 77.7548 .325 & & 2.503626 & 16.702 & -0.059 & $\mathrm{E}, \mathrm{AM}$ \\
\hline 53 & LMC_SC9 & $05134039-6918217$ & 79.5378 .261 & & 0.956442 & 16.704 & -0.088 & \\
\hline 54 & LMC_SC4 & $05263256-6945127$ & - & 1037 & 2.233238 & 16.718 & -0.023 & \\
\hline
\end{tabular}


Table 1. continued.

\begin{tabular}{|c|c|c|c|c|c|c|c|c|}
\hline \# & $\begin{array}{l}\text { OGLE } \\
\text { field(s) }\end{array}$ & $\begin{array}{l}\text { OGLE } \\
\text { name }\end{array}$ & $\begin{array}{c}\text { MACHO } \\
\text { name }\end{array}$ & $\begin{array}{l}\text { EROS } \\
\text { name }\end{array}$ & $\begin{array}{l}P_{\text {orb }} \\
\text { [d] }\end{array}$ & $\begin{array}{c}V \\
{[\mathrm{mag}]}\end{array}$ & $\begin{array}{l}V-I \\
{[\mathrm{mag}]}\end{array}$ & Remarks \\
\hline 55 & LMC_SC9 & $05130354-6917122$ & 79.5258 .87 & & 3.289306 & 16.740 & -0.055 & \\
\hline 56 & LMC_SC9 & $05143268-6912269$ & 79.5501 .310 & & 1.232396 & 16.757 & -0.091 & \\
\hline 57 & LMC_SC7 & 05174797-6904161 & - & & 3.954434 & 16.777 & 0.041 & \\
\hline 58 & LMC_SC7 & $05174804-6945493$ & 78.6097 .233 & & 5.231974 & 16.814 & -0.088 & $\mathrm{E}$ \\
\hline 59 & LMC_SC14 & $05025511-6853029$ & 1.3691 .210 & & 2.789120 & 16.821 & -0.089 & $\mathrm{E}, \mathrm{AM}$ \\
\hline 60 & LMC_SC21 & $05222806-7022407$ & 6.6814 .103 & & 5.376945 & 16.852 & 0.008 & $\mathrm{E}$ \\
\hline 61 & LMC_SC4 & $05263908-6936060$ & 77.7552 .249 & & 3.932107 & 16.852 & -0.128 & $\mathrm{E}$ \\
\hline 62 & LMC_SC17 & $05383042-6949483$ & 81.9484 .91 & & 4.261134 & 16.853 & 0.031 & $\mathrm{E}, \mathrm{AM}$ \\
\hline 63 & LMC_SC1 & 05324193-6951092 & 81.8516 .176 & & 4.954343 & 16.892 & -0.064 & $\mathrm{E}, \mathrm{AM}$ \\
\hline 64 & LMC_SC4 & 05260950-6959109 & 77.7425 .249 & & 17.366232 & 16.917 & -0.027 & $\mathrm{E}$ \\
\hline 65 & LMC_SC9 & $05132215-6927252$ & 5.5376 .2159 & & 1.788358 & 16.927 & -0.106 & \\
\hline 66 & LMC_SC6 & $05222482-6936226$ & 78.6826 .298 & 1041 & 2.688218 & 16.929 & 0.003 & E \\
\hline 67 & LMC_SC2 & $05312473-6925281$ & 77.8281 .58 & & 2.536663 & 16.944 & -0.036 & $\mathrm{E}$ \\
\hline 68 & LMC_SC11 & 05092769-6856194 & 79.4658 .4032 & & 1.827980 & 16.983 & -0.079 & \\
\hline 69 & LMC_SC3 & 05285710-6948441 & 77.7912 .282 & & 6.069953 & 16.992 & -0.044 & \\
\hline 70 & LMC_SC9 & 05142797-6854210 & 79.5505 .198 & & 9.002650 & 16.995 & 0.078 & $\mathrm{E}$ \\
\hline 71 & LMC_SC5 & $05224192-7006480$ & 6.6818 .283 & & 4.210379 & 17.001 & -0.023 & \\
\hline 72 & LMC_SC6 & $05201732-7000440$ & 6.6457 .4965 & & 2.116275 & 17.021 & -0.036 & \\
\hline 73 & LMC_SC14 & 05041689-6849509 & 1.3812 .152 & & 5.280710 & 17.029 & 0.016 & $\mathrm{E}$ \\
\hline 74 & LMC_SC9 & 05133011-6908412 & 79.5381 .199 & & 9.503056 & 17.036 & 0.018 & $\mathrm{E}$ \\
\hline 75 & LMC_SC3 & 05292500-6948081 & 77.7912 .709 & & 1.966261 & 17.067 & -0.058 & \\
\hline 76 & LMC_SC13 & $05062656-6857153$ & 1.4174 .183 & & 5.322229 & 17.094 & 0.013 & E \\
\hline 77 & LMC_SC6 & 05211299-6950512 & 78.6580 .255 & & 2.160515 & 17.107 & -0.101 & $\mathrm{E}$ \\
\hline 78 & LMC_SC10 & $05121869-6858325$ & 79.5141 .200 & & 2.390521 & 17.113 & 0.005 & $\mathrm{E}$ \\
\hline 79 & LMC_SC10 & $05122789-6920513$ & 5.5257 .3679 & & 1.982183 & 17.151 & 0.106 & \\
\hline 80 & LMC_SC9 & $05140857-6923003$ & 5.5498 .5030 & & 1.628412 & 17.164 & -0.107 & \\
\hline 81 & LMC_SC7 & $05181228-6936251$ & 78.6100 .388 & 1061 & 4.538132 & 17.182 & -0.131 & E \\
\hline 82 & LMC_SC1 & $05331282-7007025$ & 81.8512 .224 & & 5.394376 & 17.211 & -0.037 & E \\
\hline 83 & LMC_SC5/6 & $05223386-6932564$ & 78.6827 .465 & & 1.284099 & 17.224 & -0.145 & \\
\hline 84 & LMC_SC6 & $05221500-6938483$ & 78.6825 .430 & 1063 & 4.722907 & 17.226 & -0.055 & $\mathrm{E}$ \\
\hline 85 & LMC_SC6 & $05203518-6934378$ & 78.6463 .505 & & 2.117483 & 17.226 & -0.142 & $\mathrm{E}$ \\
\hline 86 & LMC_SC6 & 05222499-6938103 & 78.6825 .431 & 1053 & 3.570088 & 17.259 & -0.180 & $\mathrm{E}$ \\
\hline 87 & LMC_SC8 & 05170646-6940570 & 78.5978 .403 & & 1.635801 & 17.268 & -0.042 & \\
\hline 88 & LMC_SC10 & 05112066-6909148 & 79.5018 .187 & & 1.345239 & 17.278 & 0.035 & \\
\hline 89 & LMC_SC10 & 05115154-6920494 & 79.5136 .250 & & 1.760103 & 17.280 & -0.005 & $\mathrm{E}, \mathrm{AM}$ \\
\hline 90 & LMC_SC4 & 05264527-6944045 & 77.7550 .352 & & 6.536197 & 17.304 & -0.024 & $\mathrm{E}$ \\
\hline 91 & LMC_SC7 & $05181122-6932555$ & 78.6101 .407 & & 3.816795 & 17.317 & -0.069 & \\
\hline 92 & LMC_SC6 & 05222991-6919090 & 80.6830 .375 & & 6.310800 & 17.363 & -0.028 & $\mathrm{E}$ \\
\hline 93 & LMC_SC1 & 05335582-7019049 & 11.8630 .374 & & 4.568919 & 17.368 & 0.112 & \\
\hline 94 & LMC_SC9 & $05145023-6915416$ & 79.5621 .470 & & 4.670019 & 17.399 & -0.018 & \\
\hline 95 & LMC_SC2 & 05323120-6928535 & 81.8522 .169 & & 4.897253 & 17.431 & 0.054 & $\mathrm{E}$ \\
\hline 96 & LMC_SC7 & $05181271-6935245$ & 78.6100 .606 & 1039 & 2.575579 & 17.460 & -0.032 & E \\
\hline 97 & LMC_SC6 & $05221305-7003284$ & 78.6819 .336 & & 2.138851 & 17.466 & 0.019 & $\mathrm{E}$ \\
\hline 98 & LMC_SC14 & $05025406-6918398$ & 1.3684 .237 & & 3.825716 & 17.475 & -0.037 & \\
\hline
\end{tabular}

analyzed simultaneously by means of the improved version of the Wilson-Devinney (WD) program that includes a model atmosphere routine developed by Milone et al. (1992). The program is composed of the LC program generating light curves for assumed parameters and the differential correction program, DC. The WD program was used in two different ways: (1) to search the space of the adjustable parameters by means of the Monte-Carlo method with the LC program; (2) to get the best-fit solution for a given system with the DC one.

\subsection{Assumptions}

The DC program allows adjusting of over thirty parameters. In practice, only a few are adjusted since the remaining are either known quite well or cannot be reliably obtained from the fit. The choice of adjustable parameters have to be decided first.

Since the components of the DEBs are roughly spherical in shape, it is not possible to get the mass ratio, $q=M_{2} / M_{1}$, solely from the analysis of the light curves (Wyithe \& Wilson 2001). 


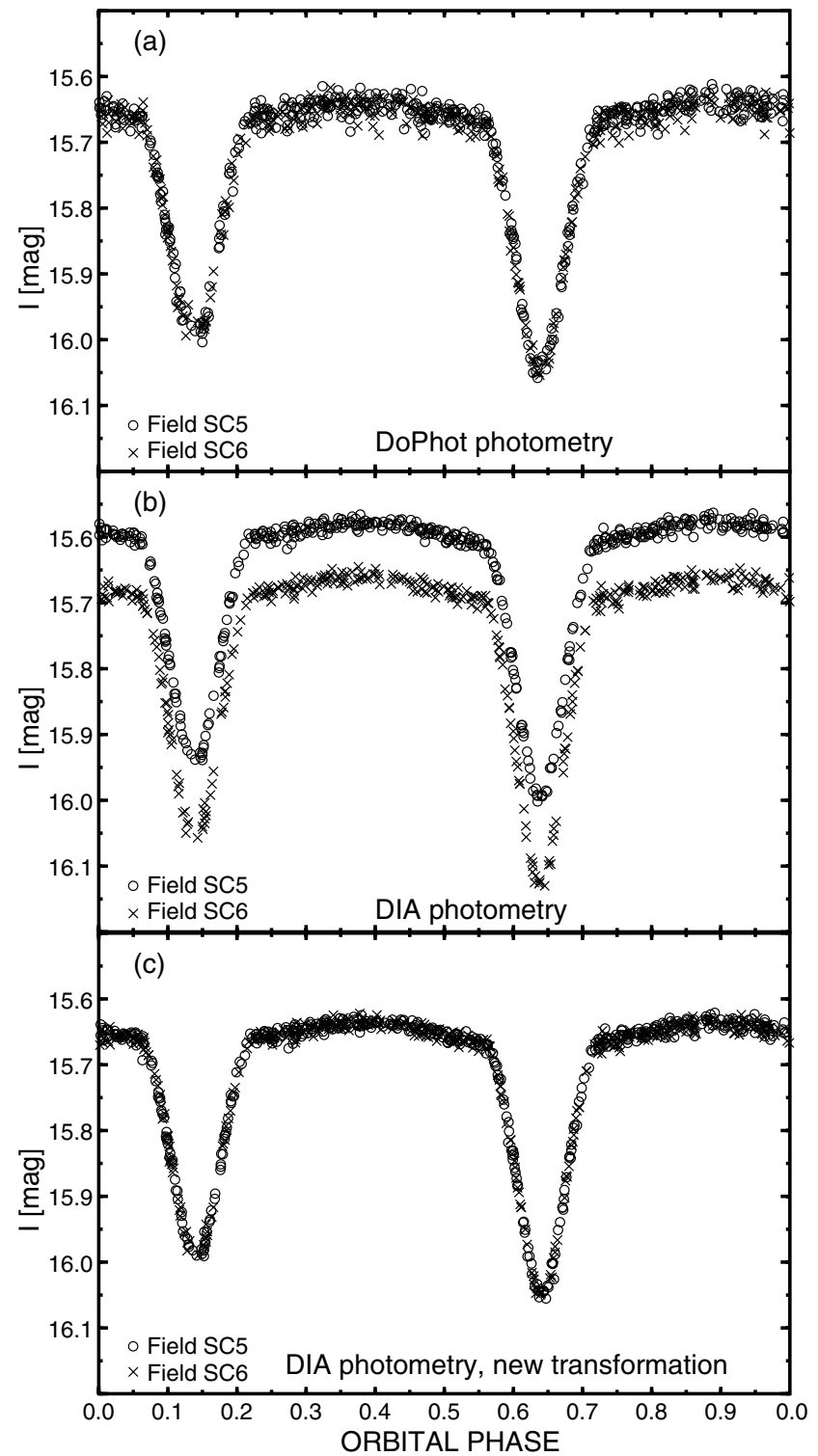

Fig. 2. $I$-filter light curves of the OGLE 05223546-6931434 (\#17) eclipsing binary observed in two fields, LMC_SC5 (circles) and LMC_SC6 (crosses). a) DoPHOT photometry, b) DIA photometry, c) DIA photometry with new transformation to magnitudes and a 0.003-mag shift between zero points applied. Note the reduction of scatter in this diagram with respect to the light curve shown in panel a).

Michalska \& Pigulski (2004) presented an example showing that equally good fits can be obtained in a very large range of mass ratios. It is therefore reasonable to assume $q=1$, as we did in this analysis, although it is obvious that for systems with unequal minima, $q$ might be far from unity. The WD program was run with detached geometry $(\mathrm{MODE}=2)$, as the selected stars were suspected to be DEBs. If, however, the gravitational potential of one of the components appeared to be close to the critical one, the system was excluded from the analysis.

The choice we made in Sect. 3 allowed us to assume safely that the components of the analyzed systems were early-type main-sequence stars. Consequently, we assumed bolometric

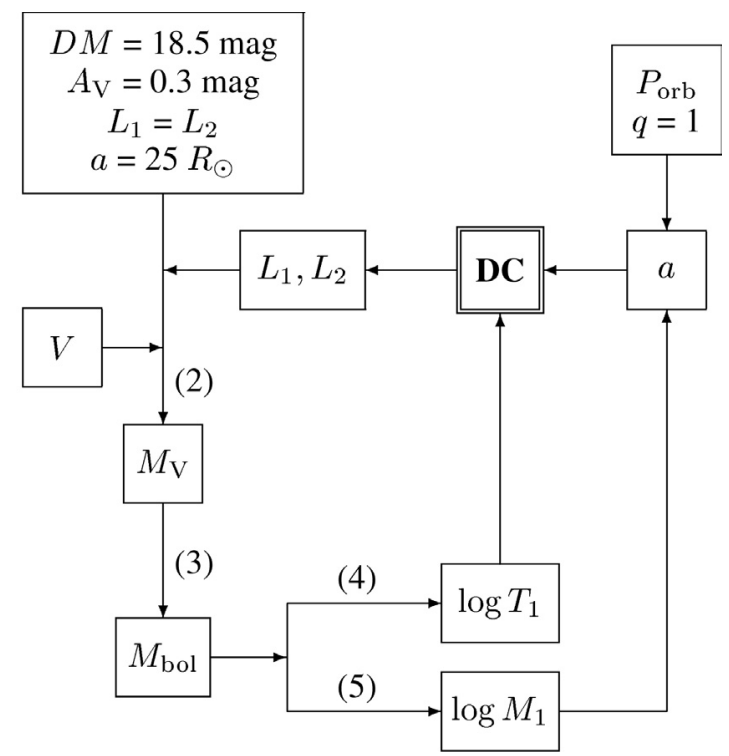

Fig. 3. The scheme of the procedure aimed in estimating the effective temperature of the primary, $T_{1}$. See Sect. 5.1 for details.

albedos and gravity darkening coefficients equal to 1.0 , a value typical for stars with radiative envelopes. The logaritmic limb darkening law $(\mathrm{LD}=2)$ with coefficients taken from van Hamme (1993) for $\log g=4.0$ was adopted. In addition, we assumed synchronous rotation and no spots. As far as the reflection effect is concerned, a detailed reflection model with two reflections (MREF $=2$ and NREF $=2$ ) was assumed for systems with circular orbits. For eccentric systems a simpler model with single reflection $(\mathrm{MREF}=1$ and $\mathrm{NREF}=1)$ was adopted. This difference is not important as the reflection effect is never significant for the systems we selected.

The major semi-axis of the relative orbit, $a$, has little to do with the light curve. However, since even an estimation gives an idea of the expected range of radial velocities, we tried to evaluate $a$. At the beginning, it was assumed to be equal to $25 R_{\odot}$. Once the masses of the components were estimated (see below), $a$ was calculated from the generalized Kepler's law.

The effective temperature of the primary component, $T_{1}$, is the last important parameter that needs to be assumed. In order to do this in a consistent way, we employed an iterative procedure shown schematically in Fig. 3. Using the out-of-eclipse $V$ magnitudes, we first estimated the absolute magnitude of the primary, $M_{\mathrm{V}}$, according to the equation:

$M_{\mathrm{V}}=V-D M-A_{\mathrm{V}}-2.5 \log \frac{L_{1}}{L_{1}+L_{2}}$,

where the average values for the LMC distance modulus, $D M=$ $18.5 \mathrm{mag}$, and total extinction, $A_{\mathrm{V}}=0.3 \mathrm{mag}$, were assumed. The component's monochromatic luminosities were first assumed to be the same, $L_{1}=L_{2}$, then they were taken from the DC output (see Fig. 3). Having calculated $M_{\mathrm{V}}$, the bolometric magnitude, $M_{\mathrm{bol}}\left(M_{\mathrm{V}}\right)$ relation in a form of a sixth-order polynomial was derived using the bolometric corrections published 
Table 2. The coefficients of the polynomials in the relations (3)-(5). $P$ is a parameter, $A$, the argument of a relation.

\begin{tabular}{rrrr}
\hline \hline Eq. & $(3)$ & $(4)$ & $(5)$ \\
$P(A)$ & $M_{\text {bol }}\left(M_{\mathrm{V}}\right)$ & $\log M_{1}\left(M_{\mathrm{bol}}\right)$ & $\log T_{1}\left(M_{\mathrm{bol}}\right)$ \\
\hline$N$ & 6 & 4 & 6 \\
$a_{0}$ & -1.46072 & 0.468944 & 4.07891 \\
$a_{1}$ & 1.67977 & $-9.46798 \times 10^{-2}$ & $-8.02904 \times 10^{-2}$ \\
$a_{2}$ & $1.94271 \times 10^{-2}$ & $-4.76657 \times 10^{-4}$ & $-1.69451 \times 10^{-3}$ \\
$a_{3}$ & $-3.00150 \times 10^{-2}$ & $-1.16486 \times 10^{-4}$ & $1.16818 \times 10^{-3}$ \\
$a_{4}$ & $-1.53159 \times 10^{-3}$ & $4.00185 \times 10^{-5}$ & $9.66154 \times 10^{-5}$ \\
$a_{5}$ & $1.07280 \times 10^{-3}$ & $\ldots$ & $-3.35212 \times 10^{-5}$ \\
$a_{6}$ & $-7.38667 \times 10^{-5}$ & $\ldots$ & $-3.48540 \times 10^{-6}$ \\
\hline
\end{tabular}

by Popper (1980). The coefficients, $a_{n}$, of the polynomial

$P(A)=\sum_{n=0}^{N} a_{n} A^{n}$,

are given in Table 2 .

The coefficients of the effective temperature vs. luminosity, $\log T_{1}\left(M_{\text {bol }}\right)$, and mass-luminosity, $\log M_{1}\left(M_{\text {bol }}\right)$ relations, are also given in Table 2. They were derived from the data published for Galactic detached main-sequence eclipsing binaries by Harmanec (1988). In order to account for smaller metallicity of the LMC, we applied a correction taken from the comparison of stellar parameters for $Z=0.02$ and $Z=0.008$ models of Bertelli et al. (1994) for the youngest stars (log(age/year) = $\left.6.6, M_{1}<15 M_{\odot}\right)$.

\subsection{Adjusted parameters}

The adjusted parameters were the following:

- phase shift, $\phi_{0}$;

- surface potentials, $\Omega_{1}$ and $\Omega_{2}$;

- effective temperature of the secondary component, $T_{2}$;

- inclination, $i$

- luminosity of the primary component, $L_{1}$.

For a few systems (\#21, \#28, \#29,\#32,\#66,\#73, \#81, and \#88) it was necessary to include into the solution the third light, $L_{3}$, as an additional adjustable parameter. The DC program provides also the fractional radii, $r_{1}$ and $r_{2}$ (back, pole, point, and side), and the luminosity of the secondary, $L_{2}$. After a first guess of the parameters, the procedure shown in Fig. 3 was repeated five times. Then, $a$ and $T_{1}$ were fixed and the iterations were repeated until the solution converged. In order to scrutinize the solution, we utilized the Monte-Carlo method (Sect. 5.3) that warrants finding a global minimum in the parameter space.

\subsection{Monte-Carlo simulations}

The Monte-Carlo (M-C) method is often used in problems with a complicated parameter space and strong correlations between parameters. It also provides an independent way to estimate the uncertainties of the parameters in the presence of the mentioned correlations.

The preliminary solution, obtained by means of the WD program, was used to define the ranges of parameters that were searched for the best solution. The parameters were the same as listed in Sect. 5.2, except for $\phi_{0}$ that was fixed at the value obtained during the DC iterations. The synthetic light curves were calculated using the LC program with a randomly-generated (within a defined range) set of parameters. The weighted sum of squares of the residuals (hereafter SSR) was stored as the goodness-of-fit estimator. At this stage we faced the problem of whether to compare the synthetic and observed light curves in all available bands and combine the resulting weighted SSRs to get a general estimation of the multi-band solution or do the same for a single band. The first approach has the advantage of being able to discriminate better solutions in case of unequal components. However, when the components are similar and there are large differences in the scatter between different bands, a single band determined the shape of the minimum in the parameter space. For that reason we decided to follow the second approach (single band), choosing the band with the smallest scatter in photometry. The band we chose is indicated in the last column of Table 3. Only in a few cases, when the scatter was similar in two bands, we did trial M-C simulations for these two bands separately and then compared the results. The global solutions obtained with the $\mathrm{M}-\mathrm{C}$ method appeared to be consistent with the preliminary ones for all but a few systems.

Some examples of the results of the M-C simulations are shown in Fig. 4 for three systems with different light-curve morphology. In this figure, we plot contours that encompass all solutions with SSR smaller than a certain value expressed in terms of the minimum value of SSR. Let us first comment on the $\Omega_{2}$ vs. $\Omega_{1}$ panels, as the surface potentials decide how well the components' dimensions are constrained. We see that $\Omega$ s are best constrained for a system with complete eclipses and components that are not similar (\#88). In the case of similar components (\#83) we can also get $\Omega \mathrm{s}$, but there are two alternative solutions. This is obvious, as the same components in a circular orbit produce eclipses indistinguishable in shape. Wyithe \& Wilson (2001) call these solutions "aliasing". For partial eclipses (\#49), the solutions spread over a crescent-shaped area that is quite wide in both $\Omega$ s. This is understandable as the change in $\Omega$ can be easily compensated by change of inclination. The relative radii for a system with partial eclipses is therefore much poorly constrained. In general, this means that such systems are much less suitable for the distance determination.

\subsection{Discussion of solutions}

Table 3 lists the parameters of 98 systems derived by means of the WD program. For 84 stars from this sample we also provide the results obtained with the $\mathrm{M}-\mathrm{C}$ simulation. The remaining fourteen eccentric systems show a detectable apsidal motion and the M-C simulation was not performed for these stars. They are discussed separately in Sect. 6. 

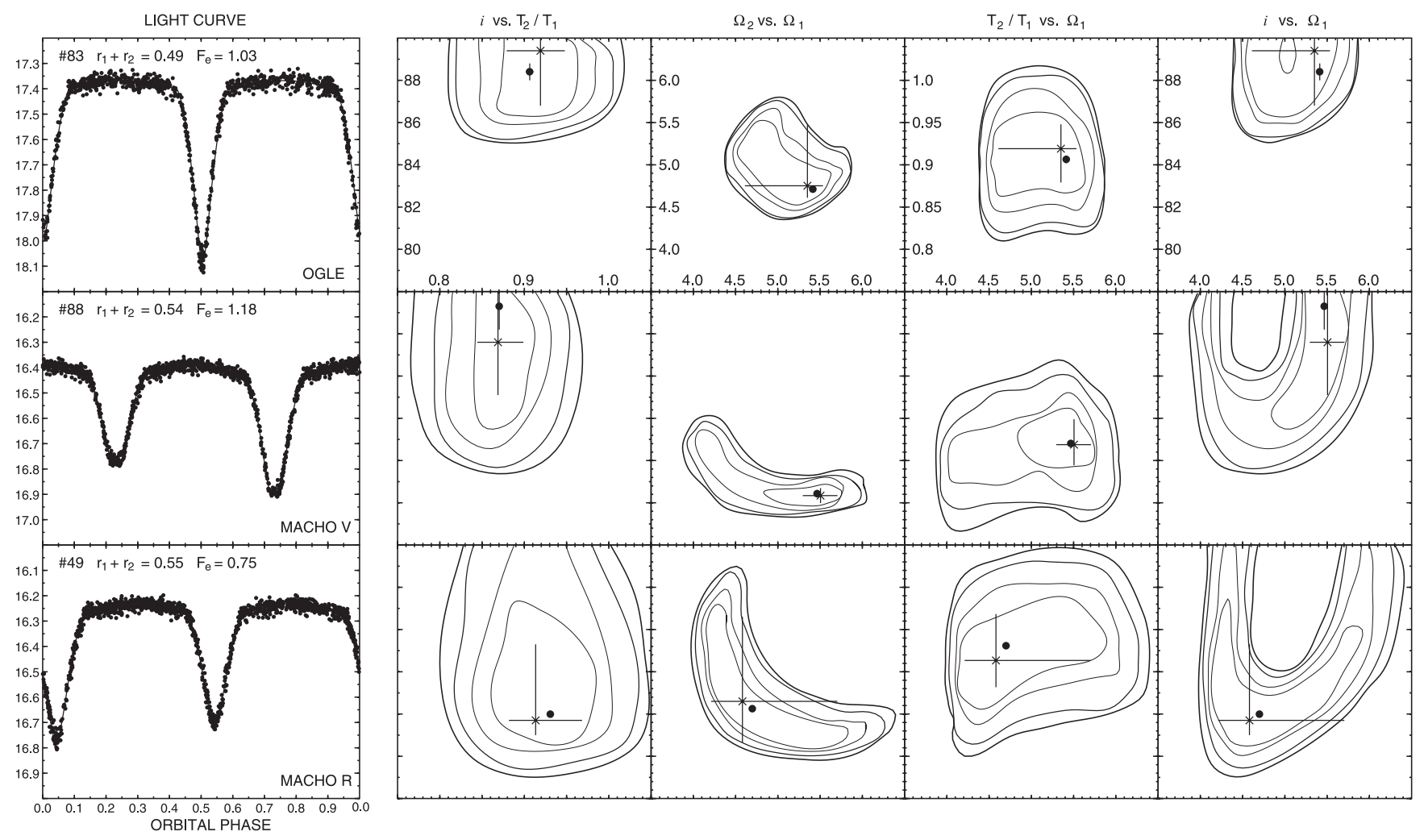

Fig. 4. Results of the M-C simulations for three DEBs with different light-curve morphology. Left: light curves of \#83 (complete eclipses, similar components), \#88 (complete eclipses, different components, and \#49 (partial eclipses). The ordinate range is the same for all three stars and equals to 1 mag. Right: panels showing the dependencies between $i, T_{2} / T_{1}, \Omega_{1}$ and $\Omega_{2}$. The ranges of the parameters are the same as labeled at the top. The contours we show encompass all solutions with SSR smaller than $A \cdot \operatorname{SSR}_{\min }$, for four values of $A: 2,1.73,1.41$, and 1.22 . The WD and M-C solutions are plotted as dots and crosses, respectively, with error bars.

Since, as shown above, surface potentials are not well constrained for the DEBs, we instead provide in Table 3 the sum of relative radii, $r_{1}+r_{2}$. In addition, the $F_{\mathrm{e}}$ parameter is given, defined by Wyithe \& Wilson (2001) as:

$F_{\mathrm{e}}=\frac{r_{1}+r_{2}-\cos i}{2 r_{2}}$,

where $r_{1}$ and $r_{2}$ denote the fractional radii (in terms of the relative distance during the eclipse) of the larger and smaller star, respectively. For systems with total eclipses, $F_{\mathrm{e}} \geq 1$. However, the denominator of Eq. (3) is not well known, so that the values of $F_{\mathrm{e}}$ we provide should be treated with caution. In the case of an eccentric orbit, the relative distance can be different in both eclipses. For this reason we list two values of $F_{\mathrm{e}}$ in Table 3. Finally, the range of radial velocities, resulting from the derived parameters, masses of the components and dimensions estimated in Sect. 5.1, are given in the tenth column of Table 3. This is a rough estimation, but it can be useful in view of the future spectroscopic observations.

The parameters of the systems are also shown in Figs. 5a-f. As a result of the application of the selection criteria (Sect. 3), we deal mostly with systems that have similar components. This can be seen in Fig. 5c: for most systems $0.8<T_{2} / T_{1}<$ 1.0, with only a few stars outside this range. From Figs. 5a and $5 \mathrm{~d}$ we see that the closest systems (i.e., having largest $r_{1}+$ $r_{2}$ ) have - as expected - mostly circular orbits and shortest periods. For $r_{1}+r_{2} \geq 0.5$ all orbits are circularized, which is consistent with the results of North \& Zahn (2003). Practically all systems with $P_{\text {orb }}$ shorter than $\sim 2 \mathrm{~d}$ have circularized orbits. This is a value typical for early-type, i.e., young systems, but much shorter than in older populations (Mathieu \& Mazeh 1988).

\section{Systems with apsidal motion}

As a consequence of the tidal and rotational distortions, the apsidal motion is observed in some eccentric systems. With the aid of theory, the knowledge of the rate of apsidal motion can be used to test the internal structure of the binary components (Claret \& Giménez 1993; Claret 1999, and references therein) or even derive their masses (Benvenuto et al. 2002). We detected that fourteen eccentric systems listed in Table 1 exhibit measurable apsidal motion. Since the WD program allows fitting of the rate of change of the longitude of periastron, $\dot{\omega}=$ $\mathrm{d} \omega / \mathrm{d} t$, we derived these values from the WD fits. They are listed in the fourth column of Table 4. The values of $e$ and $\omega$ are repeated from Table 3 for reference. Note that for systems with apsidal motion, $\omega$ is given for epoch HJD 2450500.0.

The $O-C$ diagrams for the 14 systems under consideration are shown in Fig. 6. They were obtained in the following way. First, the MACHO blue and OGLE-II data were divided into five to thirteen subsets, depending on the star. Then, the WD program was run separately for each subset with $e$ and $\omega$ fixed. The phase of primary and secondary minimum was next 

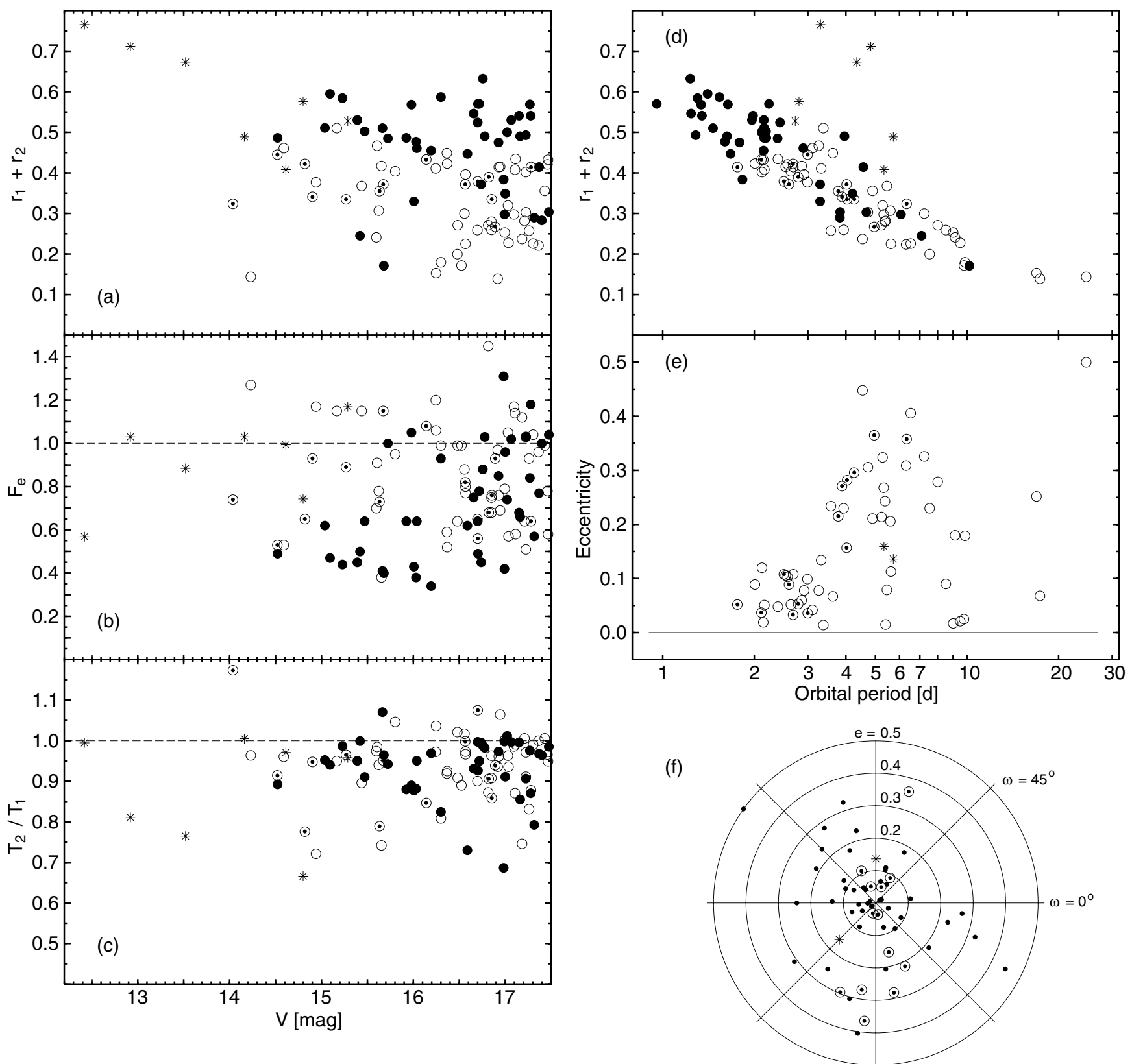

Fig. 5. Parameters of 98 systems selected in this paper. For comparison, the same parameters are also plotted (as asterisks) for the seven systems listed in the Introduction that were already used to derive the distance of the LMC. Left: sum of relative radii, $r_{1}+r_{2}$ a), $F_{\mathrm{e}}$ parameter b), and ratio of effective temperatures, $T_{2} / T_{1}$, plotted against the $V$ magnitude. Parameters for systems with circular orbits are plotted as filled circles, for eccentric systems, as open circles, and the eccentric systems with detected apsidal motion, as encircled dots. Right: sum of the relative radii, $r_{1}+r_{2} \mathbf{d}$ ), and eccentricity, $e \mathbf{e}$ ), plotted as a function of the orbital period. The same symbols as in panels a)-c) are used. The f) panel shows the $e$ vs. $\omega$ (longitude of periastron) dependence. Systems with apsidal motion are plotted as encircled dots.

derived from the fit. These phases were transformed into times of minimum closest to the mean epoch of all observations in a given subsample.

Having obtained the times of minimum from all subsets, we calculated the number of elapsed cycles, $E$, for each minimum and then fitted them with a function

$T_{\min }=T_{0}+P \times E$ deriving $T_{0}$ and $P$, separately for the primary and secondary ones. A mean of both periods, $P_{\text {mean }}=\left(P_{\text {prim }}+P_{\text {sec }}\right) / 2$, is given in Table 4 . We have also averaged the initial epochs; the mean initial epoch $T_{0 \text {, mean }}$ given in Table 4 was equal to the average of $T_{0}^{\mathrm{prim}}$ and $T_{0}^{\mathrm{sec}}+P_{\text {mean }} / 2$. Using the observed epochs, the values of $P_{\text {mean }}$ and $T_{0 \text {, mean }}$, we calculated the $O-C$ values plotted in Fig. 6, where the calculated epochs $C$ were derived from the equation

$C=T_{0, \text { mean }}+P_{\text {mean }} \times E$ 

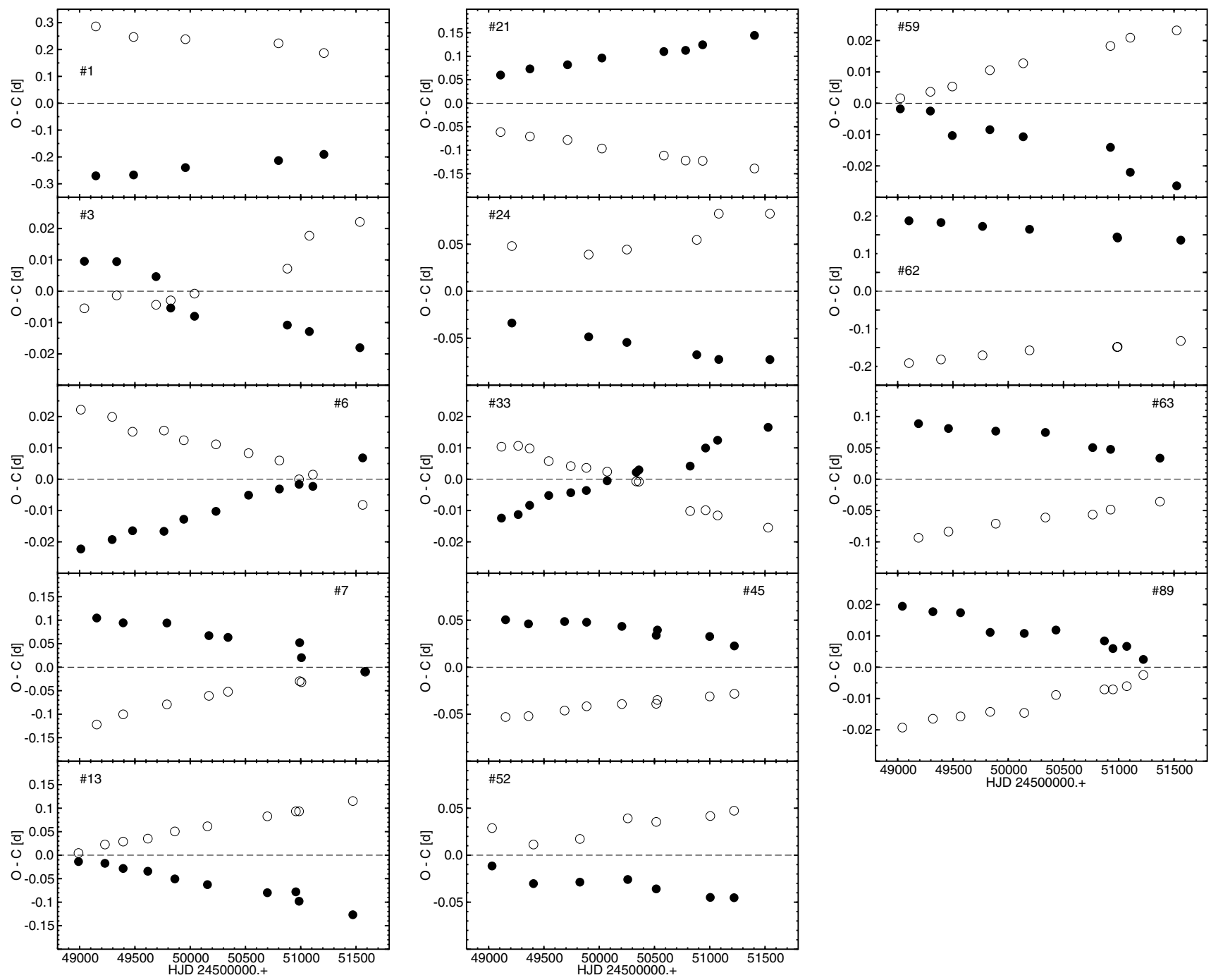

Fig. 6. The $\mathrm{O}-\mathrm{C}$ diagrams for fourteen systems with apsidal motion. The filled and open circles denote the primary and secondary times of minimum, respectively. Note different ordinate scales.

for the primary and

$C=T_{0, \text { mean }}+P_{\text {mean }} \times(E+0.5)$

for the secondary minima.

As can be seen in Fig. 5e, systems with detected apsidal motion tend to group around $\omega \sim 90^{\circ}$ and $270^{\circ}$. This is clearly an observational selection effect as in these configurations the effect on the position of the observed minima is largest. The $\dot{\omega}$ values are not determined very accurately (Table 4 ), but they can be used to estimate the apsidal periods, $U=360^{\circ} / \dot{\omega}$. The values of $U$ are generally longer than $\sim 40$ years. The lack of systems with shorter $U$ can be understood as we selected only the DEBs. In DEBs the tidal effects are not as strong as in the systems with closer components for which $U$ is sometimes very short (Petrova \& Orlov 1999).

The systems with apsidal motion we found are not the first known in the LMC. Apsidal motion was detected in HV 2274 by Watson et al. (1992) and thereafter studied by Claret (1996). It was also found in another well-studied DEB in the LMC, HV 982 (Clausen et al. 2003). In addition,
Bayne et al. (2004) recently detected this effect in the LMC system MACHO 05:36:48.7-69:17:00 = 82.9130.25.

\section{Conclusions}

We have selected 98 detached systems in the LMC that have good photometry available. They were extracted from the OGLE-II, MACHO and EROS databases and combined. For the OGLE-II data we performed transformations from fluxes to magnitudes, ensuring both small scatter and the correct range of changes. The next step towards the determination of the distance to the LMC is to obtain radial velocities for at least some of these stars. Since they all are relatively bright, this could be achieved even with 4-m class telescopes.

For all systems we derived astrophysical parameters that could be obtained from the fitting of the light-curve. They can be used to select the targets for spectroscopic observations. Comparison of the parameters of systems that were already used to derive the distance to the LMC with those we select in this paper (Fig. 5) indicates clearly that among the latter there are many systems that are suitable for this purpose. 
Table 4. Parameters for 14 systems with apsidal motion detected. The values of $e$ and $\omega$ were repeated from Table 3. The remaining parameters are explained in the text.

\begin{tabular}{ccrcll}
\hline \hline Star & $e$ & $\begin{array}{c}\omega \\
{\left[{ }^{\circ}\right]}\end{array}$ & $\begin{array}{c}\dot{\omega} \\
{[\% \text { year }]}\end{array}$ & \multicolumn{1}{c}{$\begin{array}{c}P_{\text {mean }} \\
{[\mathrm{d}]}\end{array}$} & $\begin{array}{c}T_{0, \text { mean }} \\
{[\text { HJD 244...] }}\end{array}$ \\
\hline$\# 1$ & 0.358 & 74 & $0.86 \pm 0.04$ & 6.33000 & 9146.1025 \\
$\# 3$ & 0.036 & 283 & $6.9 \pm 1.0$ & 2.995459 & 9041.9927 \\
$\# 6$ & 0.033 & 254 & $7.2 \pm 0.8$ & 2.6788316 & 9009.9189 \\
$\# 7$ & 0.271 & 261 & $2.35 \pm 0.06$ & 3.881843 & 9153.4719 \\
$\# 13$ & 0.282 & 282 & $2.35 \pm 0.04$ & 4.034834 & 8890.6211 \\
$\# 21$ & 0.215 & 295 & $3.05 \pm 0.07$ & 3.773443 & 9106.6226 \\
$\# 24$ & 0.157 & 285 & $2.05 \pm 0.10$ & 4.018700 & 9209.9996 \\
$\# 33$ & 0.037 & 280 & $8.7 \pm 0.7$ & 2.1087292 & 9114.7029 \\
$\# 45$ & 0.089 & 60 & $3.64 \pm 0.28$ & 2.5988972 & 9153.9262 \\
$\# 52$ & 0.108 & 114 & $3.31 \pm 0.23$ & 2.503626 & 9030.7418 \\
$\# 59$ & 0.053 & 106 & $3.2 \pm 0.4$ & 2.7891195 & 9022.6667 \\
$\# 62$ & 0.296 & 248 & $1.21 \pm 0.06$ & 4.261134 & 9102.1955 \\
$\# 63$ & 0.365 & 265 & $0.79 \pm 0.03$ & 4.954343 & 9187.7252 \\
$\# 89$ & 0.052 & 71 & $5.9 \pm 0.6$ & 1.7601028 & 9041.5233 \\
\hline
\end{tabular}

Special attention should be paid to the fourteen systems in which we detected apsidal motion. Fortunately, OGLE-III continues to observe the LMC, so that we can expect that they may be studied in the future in a much more detail.

Acknowledgements. This work was supported by the KBN grant No. 1 P03D 01627 . We thank Z. Kołaczkowski for providing scripts used in the selection of eclipsing variables.

\section{References}

Alard, C., \& Lupton, R. H. 1998, ApJ, 503, 325

Alcock, C., Allsman, R. A., Alves, D., et al. 1997, AJ, 114, 326

Allsman, R. A., \& Axelrod, T. S. 2001 [arXiv:astro-ph/0108444]

Alves, D. R. 2004, New Astron. Rev., 48, 659

Barnes, T. G., Evans, D. S., \& Moffett, T. J. 1978, MNRAS, 183, 285

Bayne, G. P., Tobin, W., Pritchard, J. D., Pollard, K. R., \& Albrow, M. D. 2004, MNRAS, 349, 833

Bell, S. A., Hill, G., Hilditch, R. W., et al. 1991, MNRAS, 250, 119

Bell, S. A., Hill, G., Hilditch, R. W., Clausen, J. V., \& Reynolds, A. P. 1993, MNRAS, 265, 1047

Benvenuto, O. G., Serenelli, A. M., Althaus, L. G., Barbá, R. H., \& Morrell, N. I. 2002, MNRAS, 330, 435

Bertelli, G., Bressan, A., Chiosi, C., Fagotto, F., \& Nasi, E. 1994, A\&AS, 106, 275

Claret, A. 1996, A\&A, 315, 415

Claret, A. 1999, A\&A, 350, 56

Claret, A., \& Giménez, A. 1993, A\&A, 277, 487

Clausen, J. V., Storm, J., Larsen, S. S., \& Giménez, A. 2003, A\&A, 402,509
Cole, A. A. 1998, ApJ, 500, L137

di Benedetto, G. P. 1998, A\&A, 339, 858

Fitzpatrick, E. L., Ribas, I., Guinan, E. F., et al. 2002, ApJ, 564, 260

Fitzpatrick, E. L., Ribas, I., Guinan, E. F., Maloney, F. P., \& Claret, A. 2003, ApJ, 587, 685

Graczyk, D. 2003, MNRAS, 342, 1334

Grison, P., Beaulieu, J.-P., Pritchard, J. D., et al. 1995, A\&AS, 109, 447

Groenewegen, M. A. T., \& Salaris, M. 2001, A\&A, 366, 752

Guinan, E. F., Fitzpatrick, E. L., DeWarf, L. E., et al. 1998, ApJ, 509, L21

Harmanec, P. 1988, Bull. Astron. Inst. Czech., 39, 329

Harries, T. J., Hilditch, R. W., \& Howarth, I. D. 2003, MNRAS, 339, 157

Kruszewski, A., \& Semeniuk, I. 1999, AcA, 49, 561

Mathieu, R. D., \& Mazeh, T. 1988, ApJ, 326, 256

Michalska, G., \& Pigulski, A. 2004, New Astron. Rev., 48, 719

Milone, E. F., Stagg, C. R., \& Kurucz, R. L. 1992, ApJS, 79, 123

Nelson, C. A., Cook, K. H., Popowski, P., \& Alves, D. R. 2000, AJ, 119,1205

North, P., \& Zahn, J.-P. 2003, A\&A, 405, 677

Ostrov, P. G., \& Lapasset, E. 2003, MNRAS, 338, 141

Ostrov, P. G., Lapasset, E., \& Morrell, N. I. 2000, A\&A, 356, 935

Ostrov, P. G., Morrell, N. I., \& Lapasset, E. 2001, A\&A, 377, 972

Paczyński, B. 1997, in The extragalactic Distance Scale, ed. M. Livio, M. Donahue, \& N. Panagia (Cambridge University Press), STScI Symp. Ser., 10, 273 [arXiv:astro-ph/9608094]

Petrova, A. V., \& Orlov, V. V. 1999, AJ, 117, 587

Popper, D. M. 1980, ARA\&A, 18, 115

Pritchard, J. D., Tobin, W., Clark, M., \& Guinan, E. F. 1998, MNRAS, 297, 278

Ribas, I., Guinan, E. F., Fitzpatrick, E. L., et al. 2000, ApJ, 528, 692

Ribas, I., Fitzpatrick, E. L., Maloney, F. P., Guinan, E. F., \& Udalski, A. 2002, ApJ, 574, 771

Schechter, P. L., Mateo, M., \& Saha, A. 1993, PASP, 105, 1342

Schwarzenberg-Czerny, A. 1996, ApJ, 460, L107

Udalski, A., Kubiak, M., \& Szymański, M. 1997, AcA, 47, 319

Udalski, A., Pietrzyński, G., Woźniak, P., et al. 1998a, ApJ, 509, L25

Udalski, A., Soszyński, I., Szymański, M., et al. 1998b, AcA, 48, 563

Udalski, A., Szymański, M., Kubiak, M., et al. 2000, AcA, 50, 307

van Belle, G. T. 1999, PASP, 111, 1515

van Hamme, W. 1993, AJ, 106, 2096

Watson, R. D., West, S. R. D., Tobin, W., \& Gilmore, C. 1992, MNRAS, 528, 527

Wilson, R. E. 1979, ApJ, 234, 1054

Wilson, R. E. 1990, ApJ, 356, 613

Wilson, R. E. 2004, New Astron. Rev., 48, 695

Wilson, R. E., \& Devinney, E. J. 1971, ApJ, 166, 605

Woźniak, P. R. 2000, AcA, 50, 421

Wyithe, J. S. B., \& Wilson, R. E. 2001, ApJ, 559, 260

Wyithe, J. S. B., \& Wilson, R. E. 2002, ApJ, 571, 293

Wyrzykowski, Ł., Udalski, A., Kubiak, M., et al. 2003, AcA, 53, 1 (W03)

Wyrzykowski, Ł., Udalski, A., Kubiak, M., et al. 2004, AcA, 54, 1

Żebruń, K., Soszyński, I., Woźniak, P. R., et al. 2001a, AcA, 51, 303

Żebruń, K., Soszyński, I., Woźniak, P. R., et al. 2001b, AcA, 51, 317 